\title{
The achromatic phase knife coronagraph
}

\author{
L. Abe ${ }^{1}$, F. Vakili ${ }^{1}$, and A. Boccaletti ${ }^{2}$ \\ 1 Observatoire de la Côte d'Azur, Département Fresnel, CNRS UMR 6528, 06460 Saint Vallier de Thiey, France \\ ${ }^{2}$ Collège de France, 11 place Marcelin Berthelot, 75005 Paris, France
}

Received 20 November 2000 / Accepted 11 April 2001

\begin{abstract}
We present the concept of a nulling coronagraph which theoretically attains the brightness ratio of $10^{8}$ suitable to directly detect exo-Earths in the vicinity of their parent stars. The concept is derived from the classical Foucault's optics test and Zernike's self-destructive phase contrast principle. We propose an achromatic set-up which should dramatically improve its wide band efficiency. The whole system is simulated against a numerical model which validates a formal analytical approach. The coronagraph is then compared to other concepts and its advantages and shortcomings are outlined, suggesting future laboratory developments and tests on the sky.
\end{abstract}

Key words. techniques: interferometric - stars: binaries: close - stars: planetary systems

\section{Introduction}

The amazing growth of exo-planetary detection since the discovery of 51 Peg B (Mayor \& Queloz 1995) has triggered considerable efforts for finding new methods to record the direct signal of exo-Earths shrouded by the scattered light of their parent star due to telescope optics (Marcy et al. 2000). Indeed the huge contrast ratio of $10^{9}$ to $10^{6}$ (in $\mathrm{V}$ and $N$ bands respectively) between a G-star and its orbiting exo-Earth, demands enormous dynamic range imaging possibilites that only nulling techniques, whether interferometric or coronagraphic, could attain. Unlike Lyot's mask, nulling coronagraphs offer both the angular resolution, a few tens of milli-arc-seconds (mas), and the required dynamic range to hunt exo-Earths around a statistically meaningful sample of nearby G-type stars. Pending Bracewell interferometers, e.g. Darwin (Leger et al. 1996) or TPF (Beichman et al. 1999) a large optical telescope, e.g. NGST (Mather 1997), with an embarked coronagraph seems the most likely instrument to collect photons of an exo-planet within 2010-2015 horizon. Following the original concept Gay's Achromatic Interferometric Coronagraph, AIC hereafter (Gay \& Rabbia 1996), other designs have been proposed (Roddier \& Roddier 1997) and (Rouan et al. 2000) which, unlike AIC, present inherent chromaticity limiting their net nulling efficiency over a wide spectral band.

Following our earlier work on the NGST Exo-planet Finder (Boccaletti et al. 2000), we propose hereafter a coronagraphic design which overcomes the chromatism

Send offprint requests to: L. Abe, e-mail: Lyu. Abe@obs-azur.fr problem of common coronagraphs, i.e. both the retardation and size of the phase mask. In the next section we outline the general theory of our coronagraph. Section 3 gives a generic optical set-up to overcome the chromatism problem. In Sect. 4 the conceptual design is validated by a number of numerical experiments. Finally we compare the theoretical efficiency of our coronagraph and discuss its limitations and sensitivity to various optical and operational parameters. A mathematical description of the concept is also given in the Appendix.

\section{Conceptual design}

Our coronagraph is based on Zernike's self-destructive interference concept of phase contrast (Zernike, Nobel Prize 1953) also used by Roddier (Roddier \& Roddier 1997) for their Phase Mask coronagraph (PM hereafter) and Rouan (Rouan et al. 2000) for the Four Quadrant Phase Mask (FQ-PM). PM splits the amplitude of the image plane into two parts: a core region with a $0.54 \lambda / D$ diameter shifted by $\pi$ against the rest of the image. The image is assumed an ideal Airy pattern resulting from the diffraction of a completely flat wavefront across a circular pupil. The destructive interference in the conjugate pupil plane between the two half-amplitudes with opposite signs rejects most of the energy off the pupil area. Applying an apodising mask on this pupil, about 0.8 of its geometrical size, results in a 100 times darker Airy disc in the immediately following image plane. Since the $\pi$-shift and the Airy disc size both depend on $\lambda$, PM's efficiency rapidly degrades when changing the wavelength (Guyon et al. 1999). The FQ-PM is not sensitive to the geometric size of the Airy pattern, but still has a chromatic retardation dependance. 

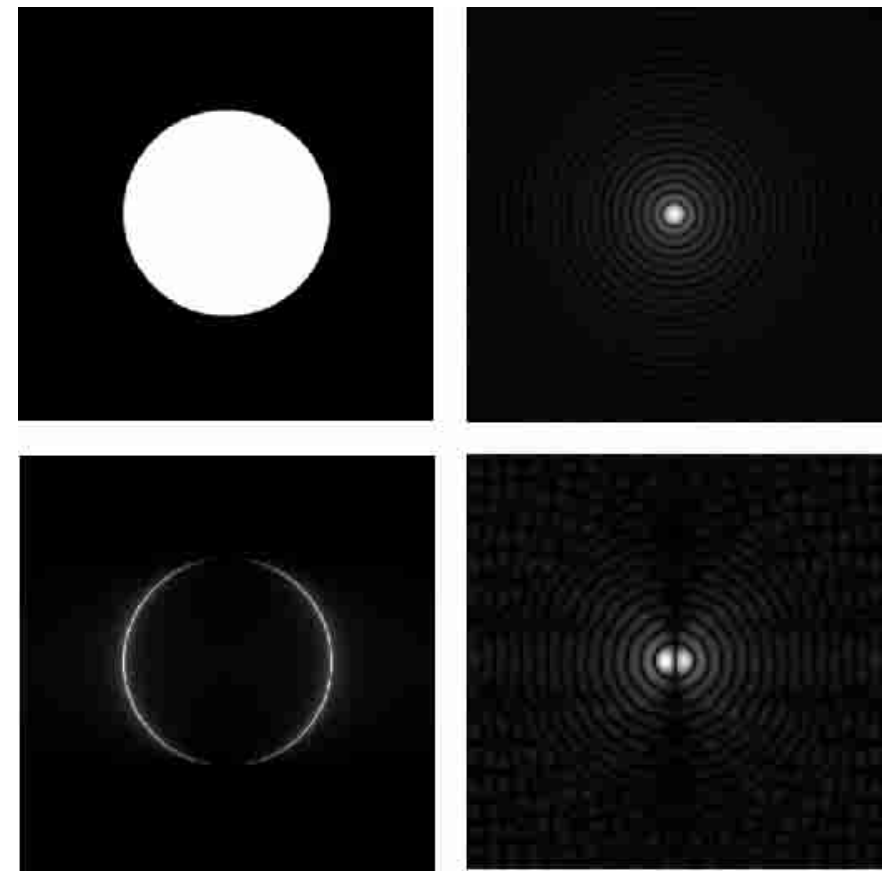

Fig. 1. Pupil intensity with perfect wavefront and its corresponding Airy pattern (top left and right). Intensity distribution after the Phase Knife Coronagraph has been applied (bottom-left): the two thin crescents encircle the pupil area perpendicular to the Knife-Edge direction. "Butterfly shape" of the point spread function of a system where half the amplitude is $\pi$-shifted in the image plane (bottom-right), and where a Lyot stop has been applied in the conjugate pupil plane of (c).

A solution for the PM chromaticity problem was originally suggested by Labeyrie (Labeyrie et al. 1999) and can also be applied to FQ-PM (Riaud et al. submitted).

For the present concept, we borrow the principle of Foucault's optics test by replacing the knife-edge by a $\pi$-shifted retarding screen (or mirror), hence the name of Phase-Knife Coronagraph (PKC hereafter). When the phase knife-edge crosses exactly the center of the Airy disc the maximum of a destructive interference occurs in the conjugate pupil image which presents two bright crescents symmetrical to the knife edge direction, as amateur mirror-polishers often observe when performing Foucault's test (Fig. 1, bottom-left). Now if we apply a circular apodizing mask on this pupil, occulting its two lateral bright crescents, we will obtain a pseudo-Airy pattern with a strong cone-like transversal extinction area (Fig. 1, bottom-right). Compared to the original input Airy pattern the intensity distribution undergoes here a noticeable nulling parallel to the phase-knife edge, but is rather ineffective in the perpendicular direction. Note that in this concept the geometrical dependence of the phase-mask is naturally eliminated. However if the image of the exoplanet happened to exactly fall along the phase-knife edge its pseudo-Airy pattern would also vanish, exactly like its parent star. Now if we apply the same operation of the phase-knife edge a second time on the output pseudo-Airy pattern in the direction perpendicular to the first phase-

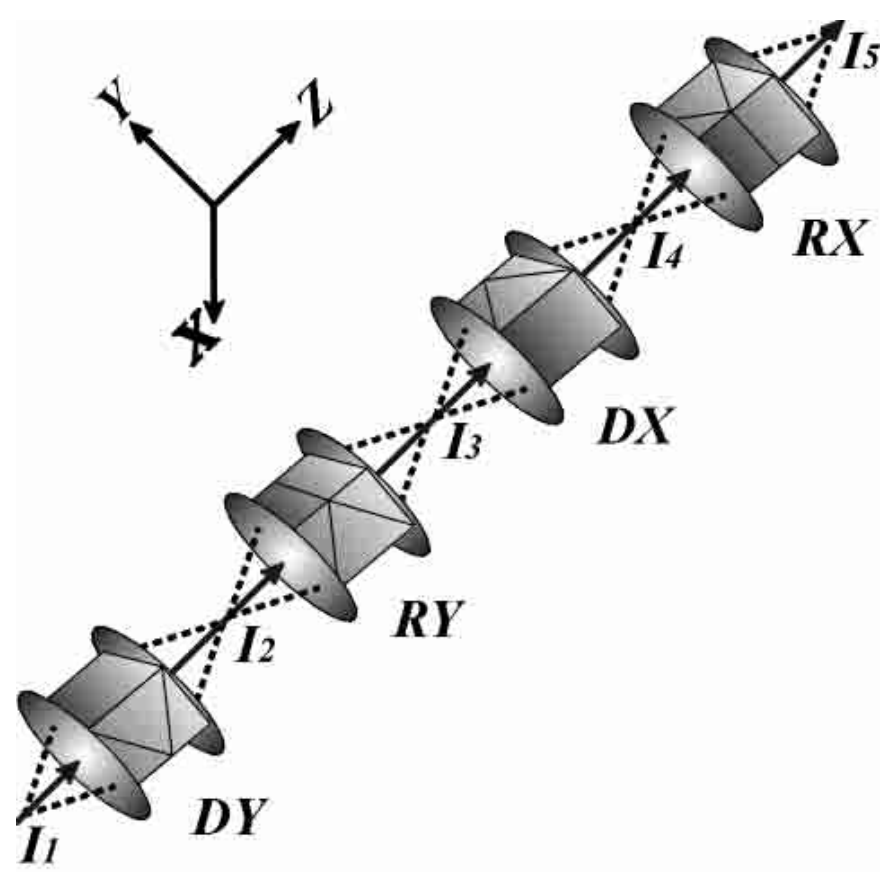

Fig. 2. Generic 3D optical scheme of the PKC. I1 is the input Airy pattern, DY is a direct vision dispersing prism in the $Y$ direction, I2 corresponds to the first chromatic phaseknife parallel to $Y$. RY is a second direct-vision prism rotated by 180 degrees with respect to DY which superimposes the dispersed phase-knived airy patterns after DY. The following DX and RX operate exactly the same as DY and RY but orthogonal to them. The final coronagraphic pseudo-Airy pattern is depicted in Fig. 3 bottom-right.

knife we obtain an extremely efficient nulling effect since the two perpendicular phase-knives cancel their mutual limb-brightening effect across the conjugate pupil. In this respect the nulling effect of the $\mathrm{PKC}$ is equivalent to the FQ-PM coronagraph (Rouan et al. 2000). However, as it will become clear in the next section, our approach offers a straightforward solution with off-the-shelf optical components to the problem of achromaticity and wide band operation of nulling coronagraphs.

\section{The achromatic PKC}

Wide band operation is crucial for any nulling interferometer or coronagraph. In this respect the AIC coronagraph (Gay \& Rabbia 1996) is the most efficient concept with the potential bonus of detecting a companion as close as its parent star's first Airy ring. AIC has two major shortcomings however: it rejects half of the incoming light (due to the beam-splitter) and its coronagraphic image becomes symmetrical (true orientations are lost). On the other hand Roddier \& Roddier's PM depends both in retardation and size on the wavelength. It conserves the absolute orientation of the image and is more efficient in terms of collected photons from the planet since it needs a less agressive Lyot pupil diaphragm. Finally the FQ-PM (Rouan et al. 2000) overcomes the geometrical problem of Roddier's phase mask but does not give a straightforward 

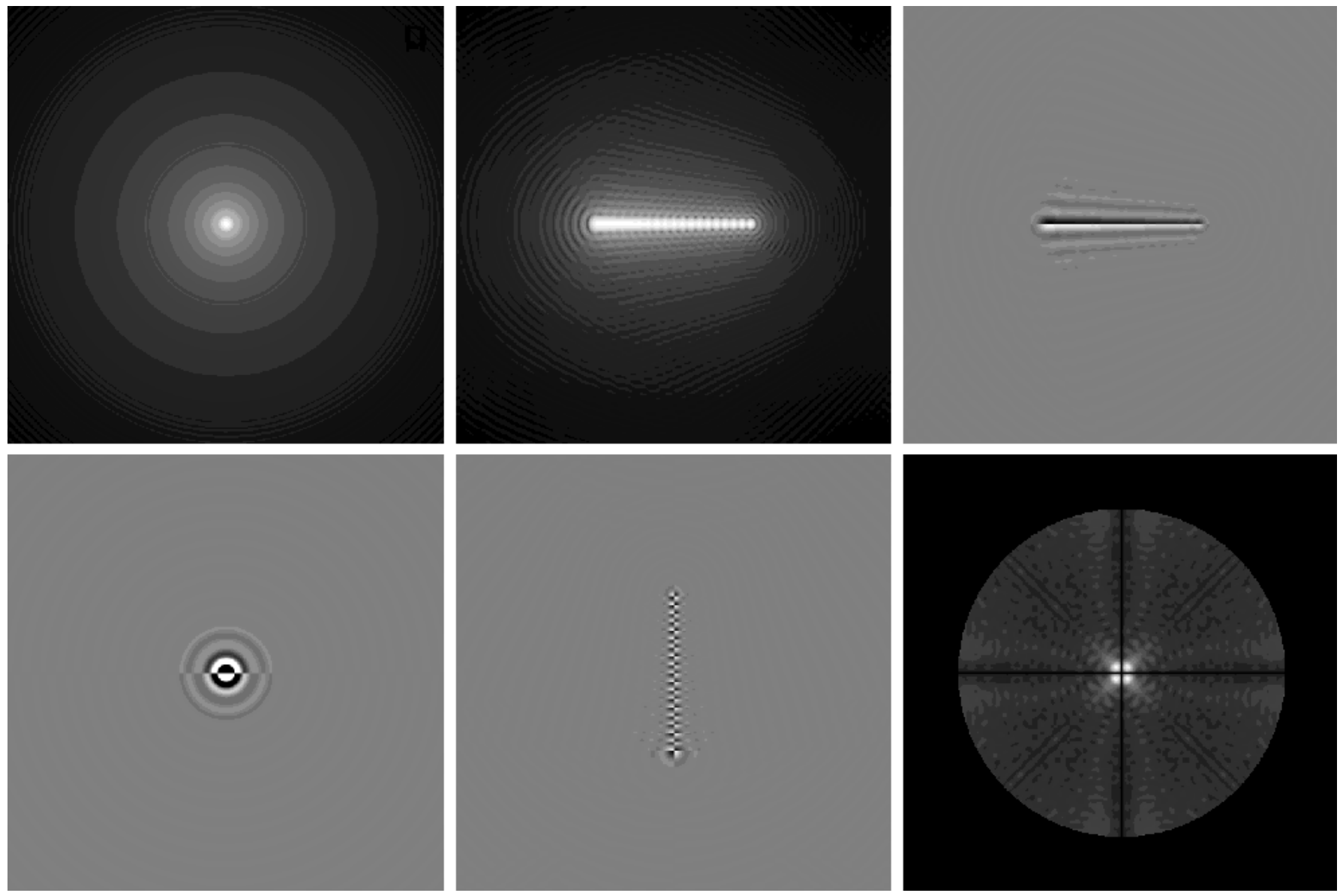

Fig. 3. Different steps of the phase-knife screen effect: (top-left) polychromatic Airy pattern (bandwidth: 400-800 nm) (corresponding to step I1 in Fig. 2), (top-middle) dispersed Airy disc, (top-right) the polychromatic phase-knife where the optical retardation follows the dispersion law (step I2), (bottom-left) an intermediate image plane where the Airy discs are de-dispersed (step I3), (bottom-middle) the polychromatic phase-knife applied in the perpendicular direction (step I4), (bottom-right) the polychromatic mutually phase-knived pseudo-Airy disc.

solution to the wavelength dependance of this phase. For the PKC, since light-cancellation is obtained in two orthogonal steps with respect to $X-Y$ directions, each step can be used to disperse the image and apply a wavelengthdependant phase retardation.

This is schematically represented in Fig. 2 where a direct vision prism (DVP hereafter) is used to disperse the input image I1 along the $Y$ axis. Here the phase retardation is produced by putting a glass plate with constant thickness on one half of the dispersed image and a thickness variable plate on the other half of the image. For the latter, the thickness follows the dispersion law (for a prism it would vary as $\lambda^{-2}$ for instance). Its gradient remains negligible along the dispersion direction, i.e. a few hundreds of nanometers for a wide band PKC, avoiding any tilt between the monochromatic wavefronts (see Sect. 5.3).

A second DVP flipped by $180^{\circ}$ around the propagation direction with respect to the first DVP re-folds the different phase-knived monochromatic images in I3 (Fig. 2). Then the operation is repeated in the perpendicular direction $X$ and finally the cross-phase-knived coronagraphic image I5 undergoes an apodising Lyot mask (not represented in Fig. 2) in the pupil plane immediately following I5 which masks the limb-brightened edges of this pupil (see Fig. A.4 top right and bottom right) to obtain the optimum starlight nulling.

Note that using two succesive DVPs for dispersing and refolding monochromatic phase-knived images is motivated by our concern to concentrate the exo-planet photons on the smallest number of detector pixels to minimize read-out noise. Once detection is positive, one could optionally remove the last DVP in order to record a low dispersion spectrum of the exo-planet for its hypothetical biomarker characterization.

\section{Numerical simulations}

\subsection{Cross dispersion effect}

In order to assess the expected performance of PKC we carried out a number of numerical experiments producing the final image (detector) plane. The model makes 
extensive use of 2D FFTs and direct convolution techniques whenever necessary. We worked on $2048 \times 2048$ double precision arrays to avoid round-out errors and aliasing effects. Figure 3 represents the different steps of the PKC nulling process. We took 20 "monochromatic" Airy discs scaled to their central wavelength from 400 to $800 \mathrm{~nm}$ by steps of $20 \mathrm{~nm}$. These monochromatic Airy discs were coadded to mimic a wide band Airy pattern (Fig. 3 top left and mid). Next, the action of the first chromatic phaseknife was applied to dispersed monochromatic Airy disc amplitudes by assuming a linear shift of the Airy pattern along the dispersion direction. The phase-knived Airy amplitudes were then folded to mimic the action of the dedispering second DVP. The action of the second chromatic phase-knife stage is mimiced by repeating the first stage in the perpendicular direction. Passing from each plane to its conjugate was performed by FFT and by adjusting the center of the diffraction patterns so as to avoid any numerical phase-artifact. Note that the Lyot stop was only applied before forming the last image by applying a diaphragm $0.9 D, D$ being the diameter of the geometrical pupil. The final coronagraphic image is shown in Fig. 3 (bottom right). We note the cross-shaped blindzone of PKC where the daugther planet PSF is affected by an intensity loss factor of about 10 (see discussion below). In order to assess the final dynamic range that one could attain with a "perfect" PKC we averaged the radial profile of the intensity in the final plane. Figure 4 depicts this profile where one can notice that even at the distance of the first Airy ring the expected contrast attains $10^{6}$. This value increases up to $10^{8}$ at the 5th Airy ring, the magic number of exo-Earth detection limit in the visible.

\subsection{Ground-based observation simulation}

As pointed out in Sect. 5, phase defects of the wavefront produced by atmospheric seeing and figuring errors of the primary mirror is a major problem for detecting faint companions. Current AO are actually unable to smooth in real time the wavefront down to the desired level of exo-planet detection.

We have carried out a numerical simulation to assess the real performance of PKC under atmospheric seeing. We generated a thousand short exposures considering an $8 \mathrm{~m}$ circular pupil including central obscuration and spiders. Compensating the wavefront turbulence with a high order $\mathrm{AO}$, we obtained an average Strehl ratio of about $70 \%$. This is typically the best performance expected for the VLT telescope using NAOS (Rousset et al. 1998). Then, we computed the coronagraphic image for each short exposure as described in Sect. 4. Finally, photon noise and readout noise were added to each frame. The long exposure image is obtained by directly co-adding the short exposures. Such an image is shown in Fig. 5. Companions as faint as $\Delta m=9$ located at the third diffraction ring, around the star, become detectable.

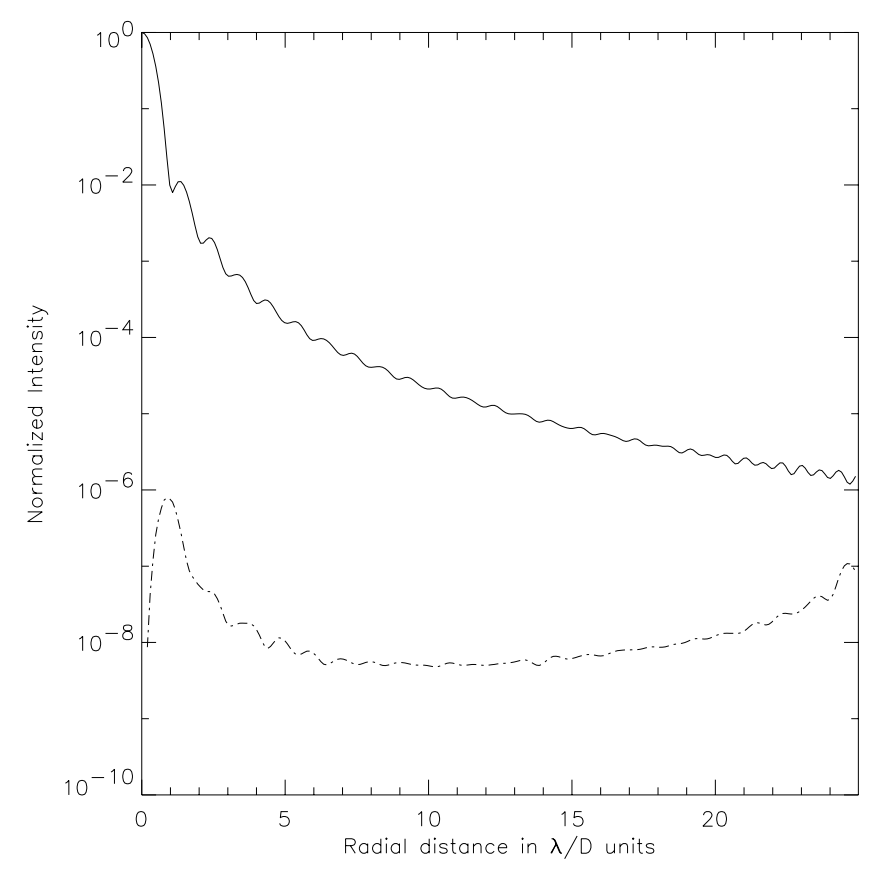

Fig. 4. Radiallly averaged profile of a polychromatic Airy pattern (solid line) obtained across a spectral bandwidth of $400 \mathrm{~nm}$ in the perfect wavefront case. The dotted line corresponds to the achromatic PKC. The maximum cancellation attains $10^{7}$ to $10^{9}$ in a large field of view at a distance of $20 \lambda / D$ from the center of the field.

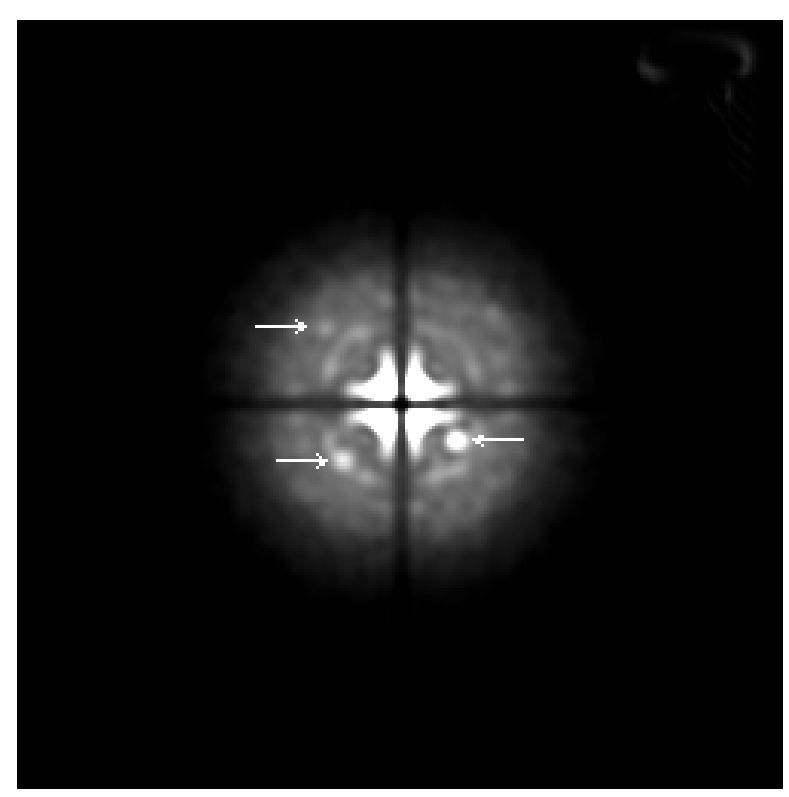

Fig. 5. Numerical simulation of $\mathrm{PKC}$ on an $8 \mathrm{~m}$ ground-based telescope equipped with adaptive optics. Atmospheric seeing and high-order compensation (Strehl ratio $\simeq 70 \%$ ) are generated with 1000 independant phase maps to provide distorted Airy patterns. Faint companions have been added to short exposures with magnitude differences of 7,8 and 9 (marked by arrows clockwise from bottom-right to top-left), with respect to the un-masked star. The image total integration time is $60 \mathrm{~s}$. Photon and read-out noise $\left(38 \mathrm{e}^{-} /\right.$pixel/frame) are added to the frames. Companions as faint as $\Delta m=9$ are directly imaged with standard integration of short frames. 


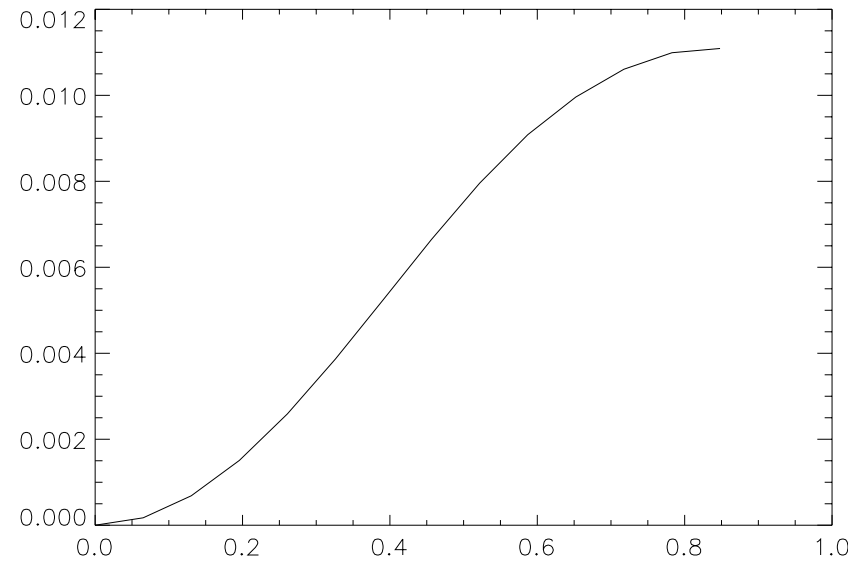

Fig. 6. The evolution of the brightness at the second diffraction ring of the coronagraphic PSF for increasing tilt errors, expressed as a fraction of the Airy radius.

\section{Discussion}

For possible applications of the PKC to the real case of space-borne telescopes it is interesting to evaluate its sensitivity to different manufacturing and operating parameters. Hereafter we limit ourselves to a few of them (startracking, residual chromatism and the inherent blind zones in PKC's accessible field of view). The effects of light scattering due to the primary mirror bumpiness and the possible angular resolution of the on-axis parent star itself will be assessed in a more detailed study in the future. The following sections describe common problems of PKC and FQ-PM (Rouan et al. 2000), the former being presented as a straightforward solution to the chromatism problem of this kind of phase mask coronagraphs.

\subsection{Global efficiency vs. centering}

Considering an ideal Airy pattern, the residual halo of the coronagraphic PSF is reduced by $10^{6}$ at the distance of the first Airy ring for a perfectly on-axis star. Tip-tilt errors represent the most degrading effects for using any coronagraphic instrument. They were simulated numerically for the PKC. The plot in Fig. 6 shows that decentering the star by $1 / 10$ th of $\lambda / D$ off-axis already drops the nulling effect to $2 \times 10^{4}$ for the second diffraction ring $(2.5 \lambda / D)$. Indeed space-borne telescopes like the NGST will be affected by much slower tip-tilt drifts, therefore meeting the severe condition given above. For other wavefront phase residuals, PKC is obviously less sensitive to centro-symmetrical aberrations.

\subsection{Detectability field}

As explained earlier, the PKC presents four inherently pseudo-blind areas along the two orthogonal directions, corresponding to the relative orientation of the two phase knives. We numerically assessed the nulling efficiency near these critical areas by computing the energy transmission for an object located on the "blind axis" and gradually de-

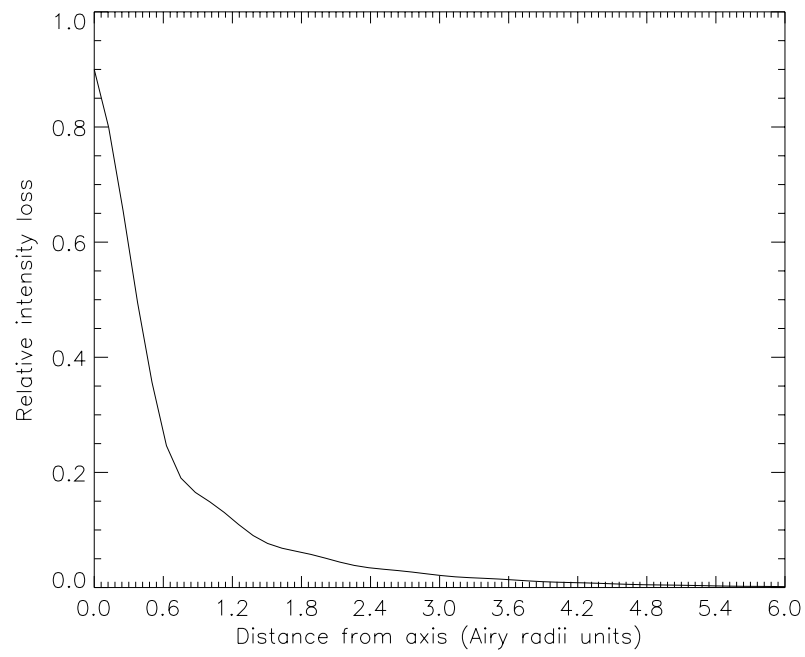

Fig. 7. Blind areas of PKC. Curve depicting the PKC effect for an object located on and near the dark axis: a 90 percent energy transmission is achieved over a 1.4 Airy radius distance perpendicularly to the axis.

viating from it. The effect, normalized on an undisturbed planet brightness is plotted in Fig. 7 where the loss is given in percentage versus shift, expressed in units of Airy radii. It exceeds 0.5 at a distance of 0.4 Airy radius. A $90 \%$ energy transmission is achieved over 1.4 unit distance. Note that on these two orthogonal axes the transmission is not zero, and some $10 \%$ intensity still pass through.

\subsection{Residual chromatism}

In sect. 3 we outlined the generic dispersing-refolding optical set-up to solve the problem of chromatism. After the dispersing step, the prismatic-shape of the phase knife must exactly match the dispersion law since a $\pi$ - retardation is only valid for a given wavelength. Indeed the higher the dispersion, the smoother the slope of the prismatic phase-knife given the $\min / \max \pi$-retardations correctly match bandwidth boundaries, even though local deviations from the ideal $\pi$-retardation may occur. We simulated these aspects by introducing slight local variations following different slopes (linear). The influence of the slope is parametrized by a $\kappa$ factor taken as the ratio of the mean Airy size to the total spectrum extent (e.g. the central monochromatic Airy disc of Fig. 3, top-middle, to the extent of the horizontal spectrum). We considered three situations where the total bandwidth $\Delta \lambda=200 \mathrm{~nm}$ (from 600 to $800 \mathrm{~nm}$ ), and $\kappa=20,5$ and 4 represented by the dotted, dash-dotted and short dashed curves of Fig. 8. In this figure the long dashed curve corresponds to a case without local phase variation, and the continuous line is the reference Airy profile without coronagraph. As a result one can see that in order to attain good nulling performances a dispersion over PSF ratio of 10 is sufficient which should easily be achieved with ordinary DVP or diffraction gratings. Note that the nulling performances of Fig. 8 


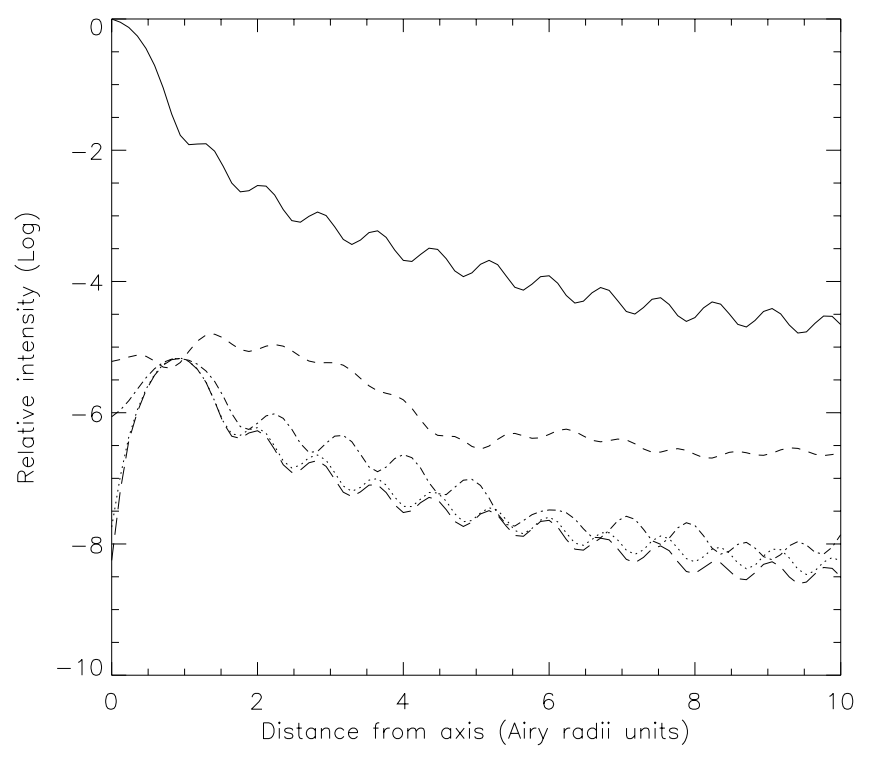

Fig. 8. Loss of nulling due to chromatism. PSF profiles for different local phase variations due to phase knives chromatic efficiency. From bottom to top, (long dashed) without phase variation $(\kappa=0)$, (dotted) $\kappa=20$, (dash dotted) $\kappa=5$, (short dashed) $\kappa=4$ and the reference PSF pattern without coronagraph (continuous).

differ from those previously presented (Fig. 4) and is only due to the use of smaller numerical arrays $(1024 \times 1024)$.

\section{Conclusion}

In this paper we described the principles of a nulling coronagraph which operates in an image plane and offers achromatic properties both in terms of image geometry and phase shift. The achromatisation is obtained using common optical components and the actual manufacturing should not be difficult. We showed from numerical experiments that in the ideal case of an aberration-free telescope PKC directly attains the required $10^{9}$ to $10^{6}$ nulling effect in the visible and IR wavelengths for direct detection of exo-planet photons. One of the major shortcoming of PKC is its partial blind cross-shaped areas, roughly two stripes $\lambda / D$ wide, parallel to the 2 successive phase- knives. If the exo-planet's image falls exactly along one of these stripes its Airy pattern is broken in two petals and attenuated by a factor of ten in intensity (see Fig. 1, bottom-right) which means these areas are semiblind. This can be overcome provided the telescope can rotate around its optical axis, by say $45^{\circ}$ for instance, and subtracting the two long exposures at each rotation angle with the extra bonus of subtracting the background noise. On the other hand the linear scheme of consecutive direct vision prisms and their relay optics for making $\mathrm{PKC}$ achromatic appears at first very sensitive to scattered light through the optical train. A workable PKC will operate off-axis with folded optics and baffling at intermediate image planes where care must be taken for extremely effi- cient coatings. Residual ghost images and scattered light will inevitably add an incoherent halo on the final coronagraphic image and limit the detectable faint planet within any reasonable integration time. A precise optical design involving ray-tracing calculations and post-processing of the collected data will be discussed in a next paper, including laboratory tests.

Another point which has not been discussed in this paper is the sensitivity of the achromatic PKC to different optical aberrations, specially light scattering due to residual mirror bumpiness at different spatial scales. This makes part of an end-to-end study that we are currently undertaking. It should be noticed however that for PKC, as for the other coronagraphs (AIC, PM and FQ-PM) the most critical issue remains the tip-tilt of the wavefront. The image jitter around the intersection of the two orthogonal phase-knives can dramatically reduce the nulling efficiency of PKC. Another critical issue is the chromatic dependance of the optical retardation along the direction of the phase-knife upon which the dispersed Airy disc of the star is formed. The manufacturing of this retardation, quadratic in the case of DVPs, needs careful design and manufacturing. An alternative solution, at the expense of luminosity, is to use diffraction gratings instead of DVPs. One could also think about a folded PKC using single diffraction gratings. A laboratory bread-board could more quantitatively define the nulling performance of PKC against other coronagraphic concepts. Such experiments are most desirable since PKC, thanks to its achromaticity (versus PM and FQ-PM) and throughput (versus AIC), appears as an attractive solution for the focal instrument of hyper-telescopes using a densified pupil (Pedretti et al. 2000).

Acknowledgements. L. Abe is grateful to R. Krawczyk and Alcatel-Space Industries/Cannes for supporting his Ph.D. fellowship. This paper benefitted from discussions with A. Labeyrie, P. Assus, L. Arnold, Y. Rabbia, E. Thomas and D. Mourard. The authors are indebted to D. Mouillet for his constructive and critical refereeing of the original manuscript.

\section{Appendix A: Mathematical formalism}

In order to assess the exact intensity distribution in the different pupil/image planes, in the following we give the mathematical expression of the PKC effect on the amplitude of the diffracted light in these planes. Since the PKC has a privileged direction, namely that of its edge, from a formal point of view, the problem can be studied in one dimension.

Now, the 2D amplitude distribution of a perfect Airy pattern has the form,

$a(r)=2 \frac{J_{1}(r)}{r}$

where:

$r=\sqrt{x^{2}+y^{2}}$

using $r, x$ and $y$ as the coordinates in the image plane. 


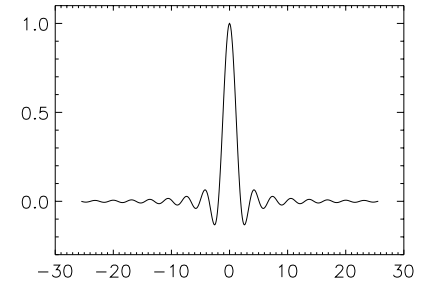

a

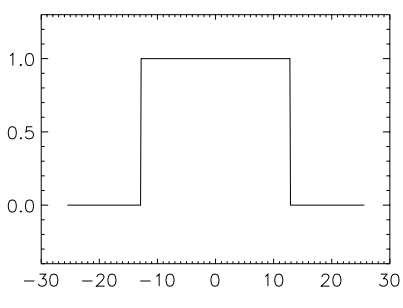

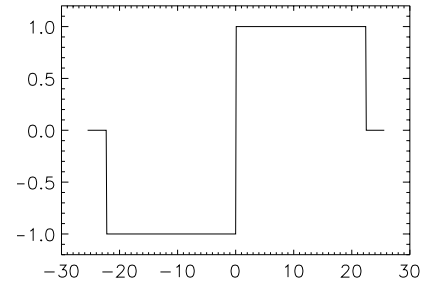

$\mathrm{b}$

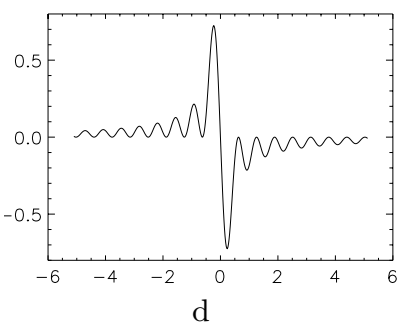

Fig. A.1. a) Amplitude profile of an Airy pattern, b) the Phase Knife function (PK) applied to the Airy pattern, c) is the Fourier transform of $\mathbf{a}$ ) and $\mathbf{d}$ ) the Fourier transform of b). To obtain the resulting amplitude in the conjugate pupil plane c) and d) functions have to be convolved. Graphics are drawn with arbitrary units.

When we want to $\pi$-shift this amplitude, $a(r)$ is multiplied by the $p k(x, y)$

$p k(x, y)=H_{x}(x, y)-H_{x}(-x, y)$

where $H_{x}(x, y)$ is the $2 \mathrm{D}$ Heaviside pseudo-function along the $x$ axis. Accordingly the resulting amplitude in the conjugate pupil plane can be expressed by Fourier transforming $a(r)$ and $p k(x, y)$ and then convolving them. We note hereafter by small letters the amplitudes in the image plane and in capital letters those on pupil planes.

From the expression of $p k(x, y)$ it can be easily seen that its Fourier transform will reduce to a one-dimensional distribution along the $u$ axis (conjugate of $x$ ). Since the Airy function is centro-symmetrical and the $p k$ function is even, the Fourier transform profiles can be written as:

$A(u)=\Pi_{\rho}(u)$

and

$P K(u)=-\frac{2 i}{u}$

$\Pi_{\rho}(u)$ is the pupil function profile of width $2 \rho$, and $P K(u)$ is a hyperbolic function which presents a singularity at $u=$ 0 . The convolution of the two functions, noted by $*$ and using $\tau$ as the variable, leads the amplitude distribution:

$F(\tau)=A * P K(\tau)=\int \Pi_{\rho}(u) P K(\tau-u) \mathrm{d} u$.

The calculation of this integral can be wisely split into three intervals. One leads to a null integral since the product of $\Pi_{\rho}$ and $P K$ is odd. The remaining parts can be
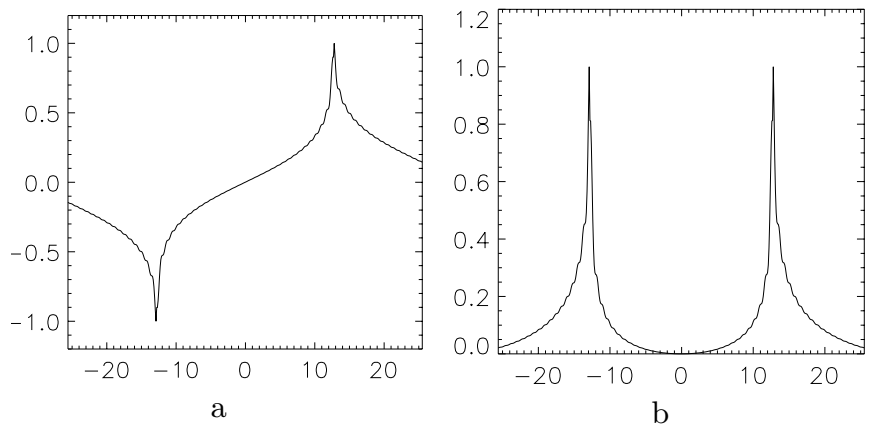

Fig. A.2. a) Amplitude profile in the pupil plane as a result of the 1-D convolution of $\tilde{P K}(u)$ with $A(u)$ and b) the corresponding intensity profile. It corresponds to the case depicted of Fig. 1. Notice that the curve near the pupil edges is not as deep as the 2-D convolution case (see below Fig. A.4c). Plots are given in arbitrary units.

classically calculated as the integral of a hyperbolic function, leading to a logarithmic type expression,

$F(\tau)=\ln (\rho+\tau)-\ln (\rho-\tau)$.

We notice that $F(\tau)$ presents two singularities on $\rho$ and $-\rho$, which correspond to the edges of the pupil. This would mean that the intensity at these points is infinite which is physically incorrect. The answer is that the $p k(x)$ distribution has finite boundaries, so we must consider a finite boundaries function, say $\tilde{p k}(x)$ then,

$\tilde{p k}(x)=H(x)-H_{x+d_{0}}(x)-H(-x)+H_{d_{0}}(-x)$

where $d_{0}$ is an arbitrary size given for the phase knife geometrical extent, which is large compared to the Airy disc. This function and its Fourier transform are plotted in Figs. A.1b and A.1d respectively, the latter being expressed as,

$\tilde{P K}(u)=i\left(-\frac{2}{u}+2 \cos (\rho u) / u\right)$.

Note that $\tilde{P K}(u)$ is odd and drops to zero for $u=0$. The convolution between the two functions $\Pi_{\rho}(u)$ and $\tilde{P K}(u)$ gives the amplitude distribution in the pupil plane (Fig. A.2).

To assess the amplitude over the whole 2D pupil plane, we need to consider one-dimensional pupil profiles for each $v$, assuming the dimensions of the phase mask are large enough compared to the airy pattern. The convolution with $\tilde{A}(u)$ (which is also one-dimensional) gives the same result as shown in Fig. A.2. This agrees completely with the 2D intensity distribution depicted in Fig. 1, bottom-left.

These expressions can be easily extended to the second $\pi$-shifting step in the orthogonal direction necessary for cross-cancelling the diffracted light in the conjugate pupil. To obtain a strong light cancellation, we have to remove the Lyot stop in the first intermediate pupil plane. 


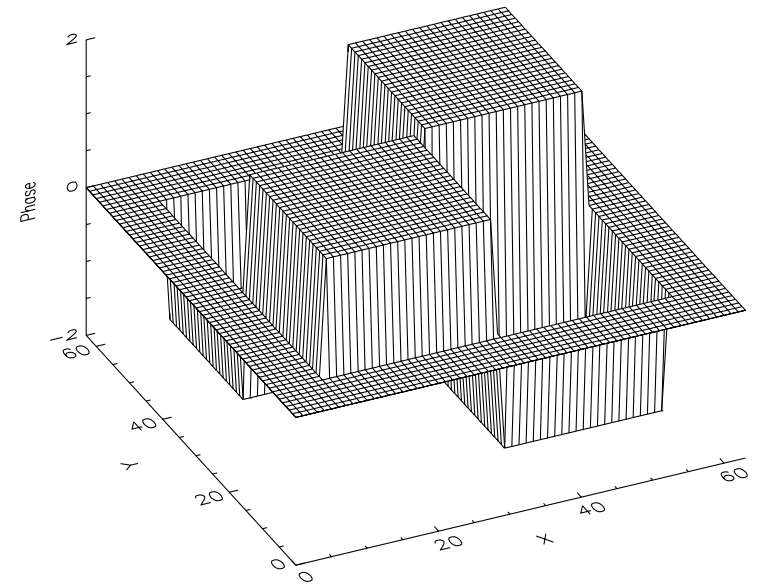

Fig. A.3. 3-D representation of the equivalent quadrant phase mask. The phase goes alternatively from $-\pi / 2$ to $+\pi / 2$.

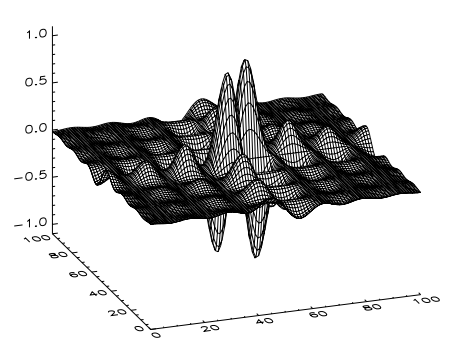

a

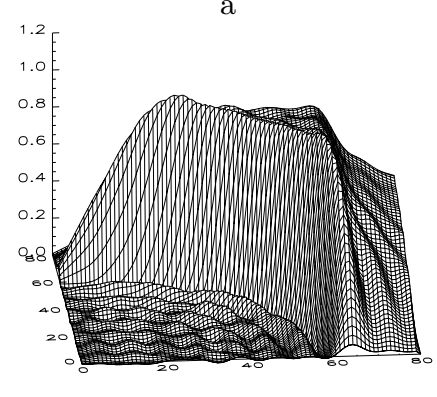

c
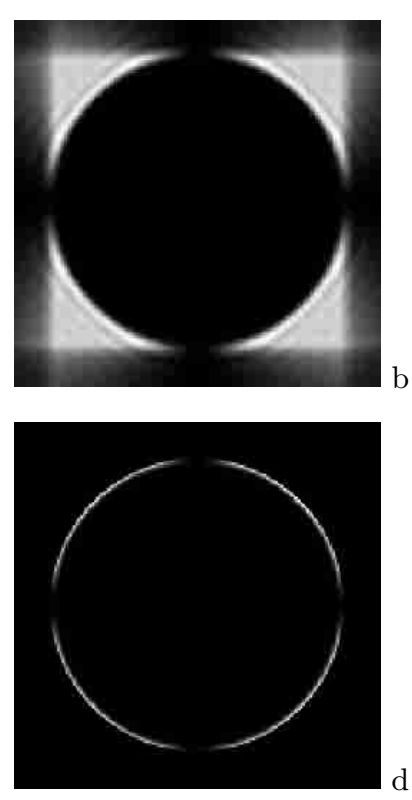

Fig. A.4. Amplitude of the Fourier transform of the equivalent quadrant phase mask a). This function is odd in a symmetry-4. When convolving this function $P K(u)$ with the pupil function $P(u)$, it contributes to a very low resulting intensity inside the pupil area b) and c). Top-right represents the modulus of the complex amplitude in the pupil plane. d) shows this modulus encircled by the pupil area. These figures were numerically computed using an exact convolution, instead of FFTs.
Indeed the physical effect of two orthogonal phase knives result in a four quadrant mask, where the phase goes alternatively from 0 and $-\pi$ or where the amplitude is alternatively left unchanged and sign-switched (Fig. A.3). The mathematical expression of the Fourier transform of the FQ-PM has been calculated and its 2-D representation is shown in Fig. A.4 top left. One can notice that this function is also odd along two orthogonal directions. Since the 2-D mathematical extension of relation A.1 has no simple expression and would require further study, we numerically computed the convolution operation using the exact mathematical formulae for the 2 -D expressions $\Pi_{\rho}$ and $\tilde{P K}$ functions (see Fig. A.4). It turns out to completely agrees with results from FFT based simulations.

\section{References}

Beichman, A., Woolf, N. J., Lindensmith, C. A. (ed.) 1999, Terrestrial Planet Finder, JPL Publication 99-3, Pasadena Boccaletti, A., Moutou, C., Abe, L., et al. 2000, NGST Science and technology exposition, ASP Conf. Ser., 207, 179

Leger, A., Mariotti, J.-M., Mennesson, B., et al. 1996, Icarus, 123, Issue 2, 249

Gay, J., \& Rabbia, Y. 1996, CR. Acad. Sci. Paris, 332, Série IIb, 665

Guyon, O., Roddier, C., Elon Graves, J., \& Roddier, F. 1999, PASP, 111, 1321

Labeyrie, A., Vakili, F., Boccaletti, A., et al. 1999, The NGST Coronagraphic Mode Study, NEF: NGST Exoplanet Finder, ESA Pre-Phase A Study Report

Marcy Geoffrey, W., \& Buttler, P. A. 2000, PASP, 112, 137

Mayor, M., \& Queloz, D. 1995, Nature, 378, 355

Mather, J. 1997, The Next Generation Space Telescope, Infrared Space Interferometry: Astrophysics \& the Study of Earth-Like Planets, ed. by C. Eiroa, et al. (Dordrecht Kluwer Academic), 227

Pedretti, E., Labeyrie, A., Arnold, L., et al. 2000, A\&AS, 147, 285

Riaud, Boccaletti, Rouan, et al. 2001, submitted to PASP

Roddier, F., \& Roddier, C. 1997, Publ. Soc. Pacific, 109, 815

Rouan, D., Riaud, P., Boccaletti, A., Clénet, Y., \& Labeyrie, A. 2000, PASP, 122, 1479

Rousset, G., Lacombe, F., Puget, P., et al. 1998, Proc. SPIE Vol. 3353, p. 508-516, Adaptive Optical System Technologies, ed. D. Bonaccini, \& R. Tyson 